\title{
Lepton-flavour violation in hadronic $\tau$ decays and $\mu-\tau$ conversion in nuclei: The role of leptoquarks
}

\author{
Kevin Monsálvez-Pozo, ${ }^{a *}{ }^{*}$ Tomáš Husek $^{b}$ and Jorge Portolés ${ }^{a}$ \\ ${ }^{a}$ Instituto de Física Corpuscular, CSIC - Universitat de València, \\ Apt. Correus 22085, E-46071 València, Spain \\ ${ }^{b}$ Department of Astronomy and Theoretical Physics, Lund University, \\ Sölvegatan 14A, SE 223-62 Lund, Sweden \\ E-mail: kevin.monsalvez@ific.uv.es, tomas.husek@thep.lu.se, \\ jorge.portoles@ific.uv.es
}

Within the Standard Model Effective Field Theory framework, with operators up to dimension 6 , we perform a model-independent analysis of the lepton-flavour-violating processes involving tau leptons. Namely, we study hadronic tau decays and $\ell-\tau$ conversion in nuclei, with $\ell=e, \mu$. Based on available experimental limits, we establish constraints on the Wilson coefficients of the operators contributing to these processes. The translation of these constraints into the most general leptoquark framework is also considered. Our work paves the way to extract the related information from Belle II and foreseen future experiments.

\footnotetext{
*** Particles and Nuclei International Conference - PANIC2021 ***

*** 5 - 10 September, $2021 * * *$

*** Online ***
}

${ }^{*}$ Speaker 


\section{Introduction}

Although both light quark and lepton families are triple replicated in nature, their description in the Standard Model (SM) of particles has a significative difference: while quark families mix, giving a rich flavour-physics phenomenology, lepton families do not. Then, one naturally wonders if the charged leptons could also experience flavour violation (CLFV). Following this reasoning, we performed in Ref. [1] a model-independent analysis of CLFV $\tau$ processes, i.e. hadronic $\tau$ decays and $\ell-\tau$ conversion in nuclei, within the framework of the Standard Model Effective Field Theory [2] (SMEFT), up to $D=6$ operators.

Among all possible BSM scenarios, a very well motivated class of theories predicts the existence of leptoquarks (LQs): electrically charged bosons of spin $S=0,1$ that couple both to leptons and quarks at the same time. We addressed the most general leptoquark scenario and applied the bounds from the CLFV analysis to constrain the LQ parameters.

\section{Calculation}

The SMEFT framework allows us to parametrize in a model-independent way, at the electroweak scale, the effects appearing from new physics living at the energy scale $\Lambda$ :

$$
\mathcal{L}_{\mathrm{SMEFT}}=\mathcal{L}_{\mathrm{SM}}+\sum_{D>4}\left(\frac{1}{\Lambda^{\mathrm{D}-4}} \sum_{i} C_{i}^{(\mathrm{D})} O_{i}^{(\mathrm{D})}\right), \quad\left[O_{i}^{(\mathrm{D})}\right]=\left[E^{\mathrm{D}}\right] .
$$

Here, while the higher energy scale $\Lambda$ usually corresponds to the masses of the heavy degrees of freedom, the Wilson coefficients (WC) $C_{i}^{(\mathrm{D})}$ encode the information of the couplings of the higher energy theory. In contrast, the operators $O_{i}^{(\mathrm{D})}$ are made out of the SM field content and contain the low-energy physics information. In this analysis we consider all $D=6$ operators driving $\tau$-related CLFV phenomena and compute the processes mentioned above. The corresponding list of operators is given in Ref. [1].

\subsection{Hadronic $\tau$ decays}

For the decays of the $\tau$ lepton into hadrons we consider three different sets: $\tau^{-} \rightarrow \ell^{-} P$, $\tau^{-} \rightarrow \ell^{-} P_{1} P_{2}$ and $\tau^{-} \rightarrow \ell^{-} V$, where $\ell=e, \mu$ and $P$ and $V$ stand for pseudoescalar and vector fields with light-quark content respectively. The computation of the width of these processes has two steps: first, the perturbative amplitudes are calculated from the $D=6$ operators of the SMEFT at the parton level; second, these partons are hadronized into the relevant final states by means of the chiral perturbation theory $(\chi P T)[3]$ and resonance chiral theory $(R \chi T)$ [4]. For this analysis we considered the experimental bounds set by Belle [5] and Belle II [6] on the following list of hadronic final states

$$
\begin{aligned}
& \tau \rightarrow \ell P: \quad P=\pi^{0}, K^{0}, \eta, \eta^{\prime}, \\
& \tau \rightarrow \ell P_{1} P_{2}: \quad P_{1} P_{2}=\pi^{+} \pi^{-}, K^{0} \bar{K}^{0}, K^{+} K^{-}, \pi^{+} K^{-}, K^{+} \pi^{-}, \\
& \tau \rightarrow \ell V: \quad V=\rho^{0}(770), \omega(782), \phi(1020), K^{* 0}(892), \bar{K}^{* 0}(892) \text {. }
\end{aligned}
$$




\section{$2.2 \quad \ell-\tau$ conversion in nuclei}

In this case, we can make use of the QCD factorization theorems to compute the total cross section of the process through the convolution of a non-perturbative part - the parton distribution functions (PDFs) - and a perturbative cross section $(\hat{\sigma})$ calculated using the $D=6$ operators of the SMEFT. Hence, the total cross section looks like

$$
\sigma_{\ell-\tau}=\hat{\sigma} \otimes f
$$

However, the nuclei are complex objects and their dynamics are not exactly those of the free partons or nucleons. We should then use the nuclear parton distribution functions (nPDFs) [7] which enconde also the nuclear effects. For the analysis we considered the experimental prospects of the NA64 experiment [8].

\section{Results}

The analysis was performed by using HEPfit [9], an open-source tool based on the Bayesian statistical framework that allowed us to translate the bounds on the aforementioned processes into constraints on the ratios $C / \Lambda_{\mathrm{CLFV}}$. The results were dominated by the Belle and Belle II bounds on $\tau$ decays. However, $\ell-\tau$ conversion in nuclei turns out to be important - once an improvement of at least two orders of magnitude in its sensitivity is achieved - in diluting the correlations among the WCs and hence, unveiling the actual origin of new physics if some of the LFV $\tau$ decays are measured.

The main results from hadronic $\tau$ decays by Belle and Belle II limits are shown in Fig. 1. The highest scale was currently probed by the dipole WC $C_{\gamma}$ giving $\Lambda_{\mathrm{CLFV}} \gtrsim 120,330 \mathrm{TeV}$ from Belle and Belle II limits respectively. Note that these results arise from the marginalized analysis where we considered all Wilson coefficients at the same time. In Figure 2 a comparison between this analysis and one considering only one WC at a time is presented. The differences stem from the strong correlations among WCs that the marginalized analysis can account for as opposed to the individual analyses.

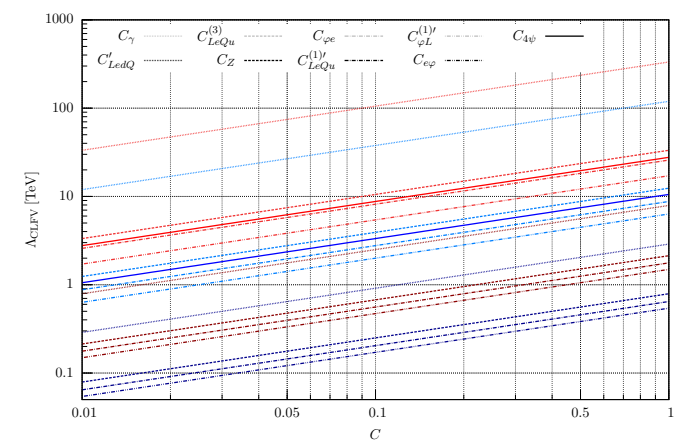

Figure 1: Constraints on $\Lambda_{\mathrm{CLFV}}$ with respect to the values of WCs, based on the current Belle (shades of blue) and expected Belle II (shades of red) limits, given at the $99.8 \%$ confidence level.

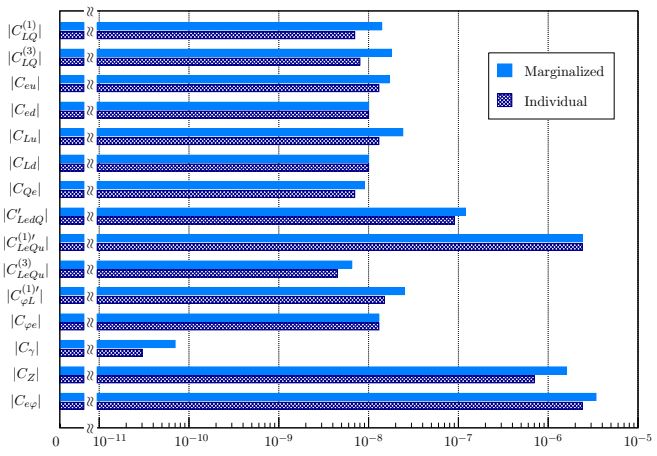

Figure 2: Constraints on $C / \Lambda_{\mathrm{CLFV}}^{2}\left[\mathrm{GeV}^{-2}\right]$ based on the current Belle limits, stemming from the marginalized and individual analyses for $\tau$ decays, given at the $99.8 \%$ confidence level. 


\section{Leptoquarks}

In the work of Ref. [10], we translate the bounds from CLFV $\tau$ processes found in Ref. [1] onto the most general leptoquark framework containing 5 scalar and 5 vectorial new fields.

\subsection{EFT matching}

In order to translate the bounds from the previous analysis, we had to compute the effective field theory of leptoquarks by integrating them away and performing the subsequent matching. Upon the integration of the LQs at leading order, we found first a straightforward matching of the fourfermion WC to pairs of Yukawa matter couplings of the leptoquarks and second, the identification $\Lambda_{\mathrm{CLFV}}=M_{L Q}$. The rest of $D=6 \mathrm{CLFV}$-related operators are obtained only at the next to leading order and they constrain the same pairs of Yukawas, then we did not consider them except for the case of the rotated $C_{\gamma}$, since it received the main constraint in the previous analysis. The vector leptoquarks cannot be unambiguously matched to gauge-boson-related operators so we only considered the scalar LQ contribution to $C_{\gamma}$.

\subsection{Results}

The matching mentioned above between the SMEFT and the leptoquark frameworks provided bounds on the ratios $y y^{\prime} / m_{S, V}^{2}$, where $y$ and $y^{\prime}$ are just symbolic notation for the different Yukawa couplings of the leptoquarks. We present those bounds for the scalar case in Table 1 (similar results were found for the vectorial case). Note that there are two pairs of Yukawas $\left(y_{2 \tau}^{\mathrm{RL}} y_{2}^{\mathrm{LR}}\right.$ and $\left.y_{1 \tau}^{\mathrm{LL}} y_{1}^{\mathrm{RR}}\right)$ which are only sensitive to $\ell-\tau$ conversion in nuclei processes. Then, only the corresponding bounds apply and these pairs of couplings are accordingly much weaker constrained. However, we found that, after the integration of the LQs, the bounds stemming from $\tau$ decays on $C_{\gamma}$ were setting a stronger constraint on these two Yukawa pairs (see bottom of Table 1).

\section{Acknowledgements}

This work has been supported in part by Grant No. MCIN/AEI/FPA2017-84445-P and by MCIN/AEI/10.13039/501100011033 Grant No. PID2020-114473GB-I00, by PROMETEO/2017/053 and PROMETEO/2021/071 (GV), and by the Swedish Research Council grants contract numbers 2016-05996 and 2019-03779.

\section{References}

[1] T. Husek, K. Monsálvez-Pozo and J. Portolés, Lepton-flavour violation in hadronic tau decays and $\mu-\tau$ conversion in nuclei, JHEP 01 (2021) 059 [2009. 10428].

[2] B. Grzadkowski, M. Iskrzynski, M. Misiak and J. Rosiek, Dimension-Six Terms in the Standard Model Lagrangian, JHEP 10 (2010) 085 [1008 . 4884].

[3] S. Weinberg, Phenomenological Lagrangians, Physica A96 (1979) 327.

[4] G. Ecker, J. Gasser, A. Pich and E. de Rafael, The Role of Resonances in Chiral Perturbation Theory, Nucl. Phys. B321 (1989) 311. 
Lepton-flavour violation in hadronic $\tau$ decays and $\mu$ - $\tau$ conversion in nuclei: The role of leptoquarks Kevin Monsálvez-Pozo

\begin{tabular}{|c|c|c|c|c|}
\hline$\tau$ decays & \multicolumn{2}{|c|}{ Bounds on $\Lambda_{\mathrm{CLFV}}[\mathrm{TeV}]$} & \multicolumn{2}{|c|}{ Bounds on Yukawas $\left[10^{-3}\right]$} \\
\hline Yukawas & Belle & Belle II & Belle & Belle II \\
\hline$\left|y_{3}^{\mathrm{LL}} y_{3 \tau}^{\mathrm{LL}}\right|$ & $\gtrsim 9.1$ & $\gtrsim 23$ & $\lesssim 12$ & $\lesssim 1.9$ \\
\hline$\left|y_{2}^{\mathrm{RL}} y_{2 \tau}^{\mathrm{RL}}\right|$ & $\gtrsim 4.6$ & $\gtrsim 14$ & $\lesssim 47$ & $\lesssim 5.0$ \\
\hline$\left|y_{2}^{\mathrm{LR}} y_{2 \tau}^{\mathrm{LR}}\right|$ & $\gtrsim 7.8$ & $\gtrsim 20$ & $\lesssim 17$ & $\lesssim 2.6$ \\
\hline$\left|y_{2}^{\mathrm{RL}} y_{2 \tau}^{\mathrm{LR}}\right|$ & $\gtrsim 6.0$ & $\gtrsim 16$ & $\lesssim 28$ & $\lesssim 3.7$ \\
\hline$\left|\tilde{y}_{2}^{\mathrm{RL}} \tilde{y}_{2 \tau}^{\mathrm{RL}}\right|,\left|\tilde{y}_{1}^{\mathrm{RR}} \tilde{y}_{1 \tau}^{\mathrm{RR}}\right|$ & $\gtrsim 7.1$ & $\gtrsim 18$ & $\lesssim 20$ & $\lesssim 3.0$ \\
\hline$\left|y_{1}^{\mathrm{LL}} y_{1 \tau}^{\mathrm{LL}}\right|$ & $\gtrsim 3.9$ & $\gtrsim 11$ & $\lesssim 64$ & $\lesssim 7.7$ \\
\hline$\left|y_{1}^{\mathrm{RR}} y_{1 \tau}^{\mathrm{RR}}\right|$ & $\gtrsim 5.4$ & $\gtrsim 16$ & $\lesssim 34$ & $\lesssim 4.1$ \\
\hline$\left|y_{1}^{\mathrm{LL}} y_{1 \tau}^{\mathrm{RR}}\right|$ & $\gtrsim 6.0$ & $\gtrsim 16$ & $\lesssim 28$ & $\lesssim 3.7$ \\
\hline$\ell-\tau$ conversion & \multicolumn{2}{|c|}{ Bounds on $\Lambda_{\mathrm{CLFV}}[\mathrm{TeV}]$} & \multicolumn{2}{|c|}{ Bounds on Yukawas $\left[10^{0}\right]$} \\
\hline Yukawas & $e-\tau$ & $\mu-\tau$ & $e-\tau$ & $\mu-\tau$ \\
\hline$\left|y_{2 \tau}^{\mathrm{RL}} y_{2}^{\mathrm{LR}}\right|$ & $\gtrsim 0.054$ & $\gtrsim 0.66$ & $\lesssim 350$ & $\lesssim 2.3$ \\
\hline$\left|y_{1 \tau}^{\mathrm{LL}} y_{1}^{\mathrm{RR}}\right|$ & $\gtrsim 0.063$ & $\gtrsim 0.75$ & $\lesssim 250$ & $\lesssim 1.8$ \\
\hline$\left(C_{\gamma} / \Lambda_{\mathrm{CLFV}}^{2}\right)^{\tau-h}$ & \multicolumn{2}{|c|}{ Bounds on $\Lambda_{\mathrm{CLFV}}[\mathrm{TeV}]$} & \multicolumn{2}{|c|}{ Bounds on Yukawas $\left[10^{-3}\right]$} \\
\hline Yukawas & Belle & Belle II & Belle & Belle II \\
\hline$\left|y_{2 \tau}^{\mathrm{RL}} y_{2}^{\mathrm{LR}}\right|$ & $\gtrsim 3.1$ & $\gtrsim 8.6$ & $\lesssim 150$ & $\lesssim 19$ \\
\hline$\left|y_{1 \tau}^{\mathrm{LL}} y_{1}^{\mathrm{RR}}\right|$ & $\gtrsim 8.2$ & $\gtrsim 23$ & $\lesssim 21$ & $\lesssim 2.7$ \\
\hline
\end{tabular}

Table 1: Obtained bounds for the scalar case. In the left-hand part of the table, we present bounds on the probed energy scale of the scalar leptoquarks mediating CLFV phenomena $\left(\Lambda_{\mathrm{CLFV}}=m_{\mathrm{S}}\right)$, considering $y y^{\prime} \approx 1$. On the right, there are bounds on the Yukawa pairs $y y^{\prime}$, assuming $m_{\mathrm{S}}=1 \mathrm{TeV}$. On the last two rows we present the bounds from the constraint on $C_{\gamma} / \Lambda_{\mathrm{CLFV}}^{2}$.

[5] HFLAV collaboration, Averages of b-hadron, c-hadron, and $\tau$-lepton properties as of 2018, 1909.12524.

[6] Belle-II collaboration, The Belle II Physics Book, PTEP 2019 (2019) 123C01 [1808.10567].

[7] K. Kovařík, A. Kusina, T. Ježo, D.B. Clark, C. Keppel, F. Lyonnet et al., ncteq15: Global analysis of nuclear parton distributions with uncertainties in the cteq framework, Phys. Rev. D 93 (2016) 085037.

[8] S. Gninenko, S. Kovalenko, S. Kuleshov, V.E. Lyubovitskij and A.S. Zhevlakov, Deep inelastic $e-\tau$ and $\mu-\tau$ conversion in the NA64 experiment at the CERN SPS, Phys. Rev. D 98 (2018) 015007 [1804.05550].

[9] J. de Blas et al., HEPfit: a Code for the Combination of Indirect and Direct Constraints on High Energy Physics Models, 1910.14012.

[10] T. Husek, K. Monsalvez-Pozo and J. Portoles, Constraints on leptoquarks from lepton-flavour-violating tau-lepton processes, 2111.06872. 